\title{
Les tentatives d'émigration par la mer de jeunes Sénégalais de Casamance
}

Migrations across the Sea of Young Senegalese from Casamance

\section{Abdoulaye Ngom}

\section{(2) OpenEdition}

\section{Journals}

Édition électronique

URL : http://journals.openedition.org/revss/353

DOI : $10.4000 /$ revss.353

ISSN : 2107-0385

\section{Éditeur}

Presses universitaires de Strasbourg

\section{Édition imprimée}

Date de publication : 15 juillet 2017

Pagination : 152-159

ISBN : 978-2-86820-969-6

ISSN : $1623-6572$

Ce document vous est offert par Université de Strasbourg

$$
\begin{array}{|l|}
\hline \text { 이모실 Les bibliothéques } \\
\hline \text { de l' Université de Strasbourg }
\end{array}
$$

Référence électronique

Abdoulaye Ngom, « Les tentatives d'émigration par la mer de jeunes Sénégalais de Casamance » Revue des sciences sociales [En ligne], 57 | 2017, mis en ligne le 15 juillet 2017, consulté le 02 mai 2019. URL : http://journals.openedition.org/revss/353; DOI : 10.4000/revss.353

Ce document a été généré automatiquement le 2 mai 2019.

Revue des sciences sociales 


\title{
Les tentatives d'émigration par la mer de jeunes Sénégalais de Casamance
}

\author{
Migrations across the Sea of Young Senegalese from Casamance
}

\author{
Abdoulaye Ngom
}

1 Le public français a pu voir récemment des scènes réelles filmées aux abords des enclaves espagnoles de Ceuta ou Melilla dans lesquelles on voit des dizaines de jeunes Africains se ruer ensemble sur des barrières de grillage de fil de fer hautes de plusieurs mètres et surmontées de guirlandes de fils barbelés, tenter de les faire ployer sous leur pression, y parvenir, les piétiner et se ruer à l'intérieur du terrain qu'elles étaient censées protéger. Ce public a compris qu'il s'agissait d'une tentative quelque peu désespérée pour ces jeunes hommes de tenter d'entrer dans "l'enclave de Ceuta» (ou de Melilla), sans nécessairement comprendre tous les enjeux de ce qu'on lui montrait. Par le présent article nous aimerions restituer, au moins en partie, le contexte de cet exemple de «pression migratoire » sur les frontières externes de l'Union européenne.

2 En tant que seules enclaves, non seulement espagnoles mais européennes, du continent africain, Ceuta et Melilla ${ }^{1}$ n'échappent pas à la pression migratoire de tout un continent. Des milliers de jeunes migrants, venus généralement d'Afrique subsaharienne, peuplent les environs de ces deux enclaves dans l'espoir de finir par trouver une occasion, un passeur, une faille, leur permettant enfin de réaliser leur rêve en pénétrant sur les terres européennes (Pian 2009). Mais comment, par quelles routes, avec quels moyens, à partir de quelles mobilisations ces jeunes Africains sont-ils arrivés jusqu'aux abords de Ceuta ou Melilla? Nous nous centrerons ici sur ceux qui viennent de Casamance ${ }^{2}$, une région relativement riche du sud du Sénégal, dont la capitale régionale est Ziguinchor. Nous y avons mené une enquête par entretiens approfondis auprès de dix-sept migrants - que l'on y appelle des candidats, terme que nous conserverons tout au long de cet article - et de leur famille. Comme il existe une grande diversité de trajectoires d'émigration, nous avons choisi de concentrer notre regard sur une « route » peu étudiée, la route de la mer. 


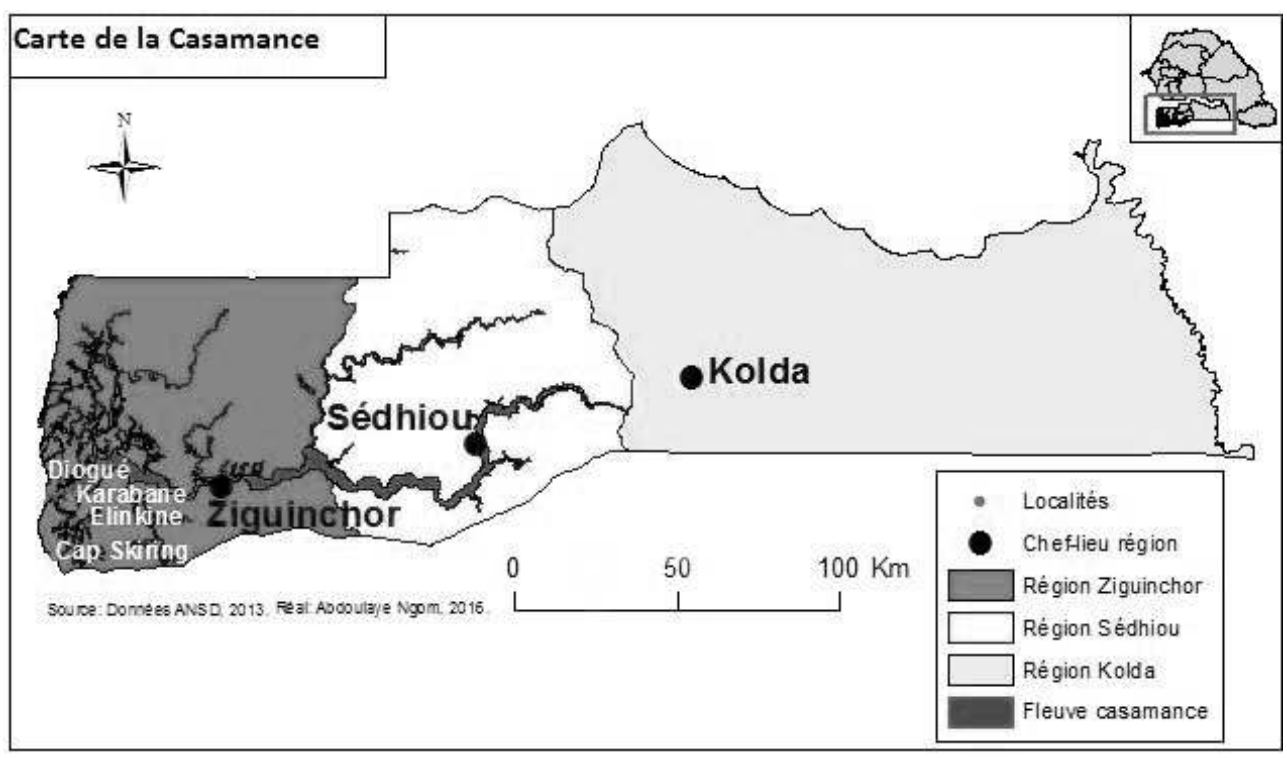

Localisation de la zone d'étude. Réalisation : A. Ngom. Source : Données provenant de l'agence nationale de la statistique et de la démographie (ANSD), 2013.

3 La Casamance est en effet une région côtière du Sénégal. Elle est située à environ $2500 \mathrm{~km}$ des enclaves de Ceuta à Melilla à vol d'oiseau. Sans visa ni argent, l'une des seules façons de se rendre de Casamance jusqu'à là-bas est de longer la côte en bateau jusqu'à Tanger. La route est longue et ce voyage prend plusieurs semaines. Il s'effectue dans des embarcations qui ne sont pas faites pour ce type de long trajet; ce sont des barques à moteur qui servent normalement à la pêche côtière, et qui sont souvent en assez mauvais état. C'est donc dans l'espoir de réussir, non seulement le voyage, mais surtout le passage sur le territoire européen, et d'accéder ainsi aux multiples opportunités qu'offre ce continent que les jeunes candidats montent à bord de ces barques, de ces pirogues, comme on les appelle localement. Qu'est-ce qui pousse les candidats à prendre la mer en direction de Ceuta et Melilla ? À la suite de quelles décisions - individuelles, familiales et collectives - et à travers quelles mobilisations? Vont-ils à la rencontre d'un mirage, éventuellement partagé par leur entourage et leur famille? Compte tenu, non seulement des risques et des sommes investies qu'il a fallu mobiliser, nous pouvons faire l'hypothèse qu'il entre dans ces décisions une bonne part de calcul rationnel. En effet ceux qui participent au financement - les parents, la famille étendue, etc. - n'ont pas d'argent à perdre et agissent donc rationnellement. Il s'agit sans doute pour eux d'un investissement, certes à haut risque, dont ils espèrent un retour : d'autres jeunes ont réussi à gagner l'Europe et renvoient au pays des sommes mensuelles qui permettent à leur famille d'améliorer leurs conditions de vie. En Casamance, une famille vit en moyenne avec trois ou quatre euros par jour, soit une centaine d'euros par mois; cela donne une idée de l'importance des « rémittences », les envois périodiques d'argent par un enfant qui aurait émigré en Europe. Après avoir discuté de la notion de clandestinité et ses dérivés, nous présenterons la Casamance, en montrant sa spécificité par rapport aux autres régions du Sénégal. Nous analyserons ensuite les déterminants (facteurs objectifs du contexte et les raisons subjectives) qui poussent les jeunes à prendre les chemins de la migration. Pour finir, nous étudierons les différents types de mobilisation de ressources qui sont investies dans ces voyages. 


\section{La clandestinité : une notion assez floue aux dérivés divers}

4 La migration clandestine peut être définie comme «une migration internationale contrevenant au cadre légal du pays d'origine, de transit ou de destination. Il y a migration clandestine soit en cas d'entrée irrégulière sur le territoire d'un État soit en cas de maintien sur le territoire d'un État au-delà de la durée de validité du titre de séjour soit encore en cas de soustraction à l'exécution d'une mesure d'éloignement" (Perruchoud 2007 : 46). Retenons ici trois cas de figure évoqués : l'entrée sur le territoire d'un État sans les documents requis permettant d'y séjourner, le maintien sur le territoire au-delà de la durée de validité d'un titre de séjour et la soustraction à une mesure d'éloignement applicable à tout individu en situation irrégulière, que l'on désigne le plus souvent comme étant des clandestins ou des sans-papiers. Le terme de clandestin ou de sans-papiers désigne tout individu qui entre, s'établit et se maintient sur le territoire d'un État, sans aucun document lui permettant d'y séjourner. Ces deux notions s'appliquent aussi à tout individu ayant effectué une demande de régularisation de sa situation auprès des autorités compétentes, mais qui s'est soldée par un échec. Tant qu'ils n'auront pas été régularisés, ils doivent éviter tout contrôle d'identité et chercher à ne pas attirer l'attention sur eux, parce que s'ils sont arrêtés, ils risquent d'être expulsés hors du territoire. Afin d'éviter une telle situation, ils développent diverses stratégies que Godfried Engbersen résume en quatre points : la mobilisation du capital social, le mariage à but résidentiel, les manipulations de l'identité personnelle, et la manifestation de prudence dans l'espace public (Engbersen 1999). Quelle que soit l'appellation employée (clandestin ou sans-papiers), il s'agit d'individus en situation irrégulière parce qu'ils ont pénétré et séjourné illégalement sur le territoire d'un État alors qu'ils n'étaient pas en possession de document (absence de visa, de titre de séjour) leur autorisant l'entrée et le maintien sur ce territoire soit dans une intention de transit ou d'installation. L'image du clandestin renvoie aussi à toute personne qui avait un permis de séjour (quels que soit la durée et le motif de sa présence) et dont le renouvellement lui a été refusé pour diverses raisons d'où l'appellation de sans-papiers qui reste péjorative et émaillée de connotations et de sens divers. Dans le cas d'étude présenté dans cet article, nous nous intéressons aux individus n'ayant aucun document officiel en leur possession qui leur permettrait d'entrer et de séjourner sur le territoire d'un État quelconque, quel que soit le motif de leur voyage. Ce sont donc des futurs clandestins sénégalais qui, chaque jour, tentent de rejoindre les côtes européennes.

\section{Présentation du cadre de l'étude}

Située dans la région du sud du Sénégal avec une superficie de plus de $28000 \mathrm{~km}$ carrés, la Casamance regroupe trois entités administratives: Ziguinchor, Kolda et Sédhiou. Les Diolas constituent la principale ethnie présente dans cette région, mais y vivent également des Peulhs, des Mandingues, des Manjacks, des Sérers, des Poulars, des Wolofs, etc. Des ressortissants de la Guinée, de la Guinée Bissau, du Ghana et du Burkina Faso sont présents aussi dans le village d'Elinkine et l'île de Djogué, en raison du développement des activités de pêche. Le climat y est de type soudanien avec une longue saison des pluies, ce qui favorise la fertilité des sols, et donc le développement de l'agriculture, de la 
riziculture, des cultures maraîchères, etc. De ce fait, la Casamance est considérée comme étant l'un des "greniers du Sénégal ». Au-delà du fait de favoriser le développement d'activités économiques, cette nature constitue en outre, un lieu symbolique donnant lieu à des rites très importants pour la vie des communautés locales. Par exemple, des rites de socialisation tels que le boukout ${ }^{3}$ et le kankourang ${ }^{4}$ donnent à la Casamance toute sa richesse culturelle.

\section{Déterminants des migrations en Casamance}

Plusieurs facteurs permettent d'expliquer les migrations clandestines à partir de la Casamance. D'une part nous pouvons citer les facteurs objectifs : le conflit armé présent dans la région, le chômage, la pauvreté, les conditions de vie difficile des ménages, les difficultés d'obtention de visa, l'influence des immigrés revenus au pays et l'impact des nouvelles technologies de l'information et de la communication. D'autre part nous retrouvons également des facteurs plus subjectifs, qui sont le désir de nombreux jeunes de «se réaliser ", leur désespoir et l'image qu'ils ont de la vie en Europe. Cette distinction fondamentale des déterminants permet de dégager ainsi plusieurs profils de candidats, ce qui montre que les motivations ou les raisons d'agir (Bourdieu 1994) varient d'un individu à un autre.

\section{La Casamance : une région connaissant un conflit armé depuis 1982}

7 La Casamance est secouée depuis plusieurs années par un conflit opposant le Mouvement séparatiste des forces démocratiques de la Casamance (MDFC) à l'armée sénégalaise. Les origines du conflit remontent à décembre 1982 (Marut 2010), suite à une marche pacifique organisée par le MDFC en direction de la gouvernance de Ziguinchor. En effet, cette marche a été réprimée de manière sanglante par les autorités sénégalaises, qui ont également procédé à de nombreuses arrestations, comme celle du dirigeant du mouvement de l'époque, l'abbé Augustin Diamacoune Senghor. Les sympathisants et partisans du mouvement se sont par la suite cachés dans la forêt de cette région afin de mettre un mouvement de rébellion pour faire sécession avec le Sénégal et revendiquer l'indépendance de la Casamance. Armés au début de coupe-coupe, de lances et de fusils artisanaux, les rebelles se sont au fil des années dotés d'armes et d'une artillerie qui leur permettent de mener des combats contre l'armée sénégalaise présente dans la zone et de lui tenir tête.

8 Le MDFC regroupe plusieurs chefs rebelles, qui revendiquent chacun leur autonomie. Pendant plusieurs années le principal dirigeant était l'abbé Augustin Diamcoune Senghor jusqu'à sa mort le 17 janvier 2007. Figure de proue du mouvement, emprisonné à deux reprises pour ses activités de propagande politique en faveur de l'indépendance de la Casamance, il fut le premier secrétaire général du MDFC. Il a été le signataire de la plupart des accords de paix conclu entre le mouvement et le gouvernement du Sénégal. Il a passé les dernières années de sa vie à tenter d'unifier le mouvement pour que les accords de paix signés puissent être respectés afin de ramener la paix en Casamance. Une autre figure charismatique du MDFC est incarnée par Salif Sadio, connu pour ses prises de position drastiques et virulentes. Il a ainsi toujours cherché à faire comprendre à l'opinion publique que les efforts pour que la paix revienne en Casamance ne pouvaient 
aboutir sans lui et ses hommes. Dirigeant une faction très importante du mouvement dans le nord de la région, César Atou Badiate est le commandant des troupes armées et représente le véritable chef de guerre du MDFC. Il est à l'origine de la plupart des exactions et des attaques orchestrées par mouvement dans la région. Enfin, le secrétaire général, Mamadou Nkrumah Sané, sous le coup mandat d'arrêt international depuis 1997, est exilé à Paris depuis plus de vingt ans. Il dirige l'aile extérieure du MDFC et s'est autoproclamé secrétaire général du mouvement à la mort d'Augustin Diamacoune Senghor sans le consentement des autres chefs rebelles.

9 En raison toutefois des divergences qui opposent les différents chefs rebelles, le MFDC est fragmenté depuis plusieurs années. En effet l'un des principaux chefs de guerre qui dirige une faction du mouvement séparatiste dans le nord de la région, Salif Sadio, a vu sa domination être contestée par les autres chefs rebelles, en particulier César Atou Badiate, qui dirige l'aile Sud du mouvement. Dans le même temps, l'autorité de Mamadou Nkrumah Sané est remise en cause par Salif Sadio. L'examen de ces rivalités internes montre les dissensions qui existent entre les différents chefs rebelles, ainsi que le caractère fragmenté du MFDC, en particulier depuis la mort de son premier et principal dirigeant, Augustin Diamcoune Senghor. À l'instar de tous les autres chefs rebelles, Mamadou Nkrumah Sané revendique depuis le début du conflit l'indépendance de la Casamance. En règle générale, les chefs rebelles cherchent tous à prendre la tête du MDFC ce qui explique pourquoi à chaque fois que l'un d'eux négocie avec le gouvernement sénégalais, l'autre partie rejette l'accord trouvé.

Depuis le début du conflit, le gouvernement du Sénégal cherche à rétablir la paix, notamment en signant plusieurs cessez-le-feu ${ }^{5}$ et accords de paix avec le mouvement séparatiste. L'accord de paix le plus récent a été signé le 30 décembre 2004. Ces accords avaient certes permis d'installer une certaine stabilité et d'instaurer la paix dans la région mais jamais de manière définitive puisque quelques mois après les combats ont repris de plus belle entre le MFDC et l'armée sénégalaise qui installe donc une situation de ni guerre, ni paix qui prévaut dans la région. Au-delà de tous ces accords de paix, il convient de souligner que la résolution du conflit en Casamance passe inéluctablement par des négociations avec l'ensemble des factions du MDFC, ce qui permettrait de trouver un consensus entre tous les chefs rebelles, afin que la paix revienne définitivement dans cette région du sud du Sénégal.

\section{Chômage et pauvreté dans les familles}

$11 \mathrm{Vu}$ la quasi-inexistence de progrès économiques malgré les efforts consentis par le gouvernement sénégalais, la persistance et l'augmentation du chômage d'année en année constitue un problème majeur en Casamance. En conséquence, la nécessité d'insérer le chômage au cœur des préoccupations des autorités s'avère plus que jamais nécessaire. Les difficultés d'accès au marché de l'emploi persistent, avec une très forte concurrence dans presque tous les secteurs d'activité. Cette tendance est aussi observable dans les autres régions du pays où les individus éprouvent d'énormes difficultés à trouver un emploi, en particulier dans la fonction publique, puisque la demande y est très largement supérieure à l'offre. Dans ce contexte, la plupart des jeunes s'orientent vers des activités journalières représentant une source de revenu. L'auto-entreprenariat connaît de fait un essor considérable depuis quelques années dans plusieurs secteurs d'activité, notamment dans le commerce. Les cireurs de chaussures, laveurs de voitures, marchands ambulants sont 
de plus en plus en nombreux dans les villes. Dans tous les quartiers de la région, le développement d'ateliers de mécanique, de menuiserie, d'artisanat, reflète le manque d'opportunités professionnelles pour les jeunes. Faible revenu, précarité et risques liés au métier exercé caractérisent ces activités de survie. Même si ces activités permettent aux jeunes de gagner de l'argent, il n'en demeure pas moins que ces ressources sont insuffisantes pour qu'ils subviennent à leurs besoins et à ceux de leur famille. C'est l'une des raisons pour lesquelles certains parents mobilisent une bonne partie de leurs ressources afin de financer les projets migratoires de leur enfant, malgré les dangers considérables que présente l'émigration (il n'y a bien souvent qu'un seul des enfants par famille qui émigre). Du fait de cette situation économique et sociale extrêmement précaire, notamment pour les jeunes de cette région, l'émigration s'impose comme étant l'alternative la plus crédible vers la réussite, peu importent les risques.

Vous voyez bien le quartier dans lequel j'habite. Les personnes vivent de façon très précaire. Les murs des maisons sont en banco ${ }^{6}$ et nous avons des problèmes d'approvisionnement en eau. Les gens sont contraints d'aller puiser de l'eau dans un puits collectif alors que d'autres vont en acheter dans cette ruelle. Je croise tout le temps des mères de famille qui sollicitent mon aide pour préparer le repas ou pour acheter des médicaments pour leur enfant malade. Je vois mes sœurs se prostituer clandestinement la nuit dans leur chambre avec pour seul éclairage une bougie. Certains jeunes vont cueillir des mangues et d'autres fruits dans la forêt pour les revendre au marché ou en faisant du porte à porte alors que d'autres sont des marchands ambulants qui travaillent pour des grossistes qui leur donnent des marchandises qu'ils vont écouler pour avoir une petite contrepartie. Tout ceci pour vous montrer que la situation va très mal et devient de plus en plus difficile et insupportable. Alors vous comprenez pourquoi nous prenons les $\operatorname{loccos}^{7}$ pour quitter ce pays où les riches sont toujours riches et les pauvres toujours très pauvres.

En Casamance, certaines familles vivent dans une pauvreté chronique, caractérisée par des habitats précaires et des conditions de vie difficiles. Cette pauvreté, observable dans plusieurs quartiers en périphérie des villes, est encore plus marquée dans les zones rurales, où les activités agricoles ne permettent plus aux familles d'assurer leur subsistance. Les familles sont frappées de plein fouet par la conjoncture économique qui accentue leurs difficultés, car l'agriculture, qui constitue la principale activité des populations rurales, est un secteur en grande difficulté depuis quelques années. Parallèlement, les problèmes d'approvisionnement en eau et en électricité, l'absence d'usine et d'entreprise, la vétusté et le caractère non praticable des routes, le manque d'infrastructures sanitaires, etc., rendent de plus en plus difficiles les conditions de vie des populations. Dans de nombreux villages, par exemple, les femmes sont contraintes de faire des dizaines de kilomètres chaque jour pour aller chercher de l'eau. De plus, en raison de l'absence de structures sanitaires, les femmes qui arrivent au terme de leur grossesse sont le plus souvent acheminées ipso facto sur des charrettes, vers des hôpitaux. Cette situation favorise, d'une part, un exode important vers les villes et une urbanisation sauvage. D'autre part, elle constitue un facteur puissant qui pousse de nombreux jeunes à embarquer sur une pirogue pour tenter de partir à l'étranger. La pauvreté est ainsi identifiée comme l'un des éléments déterminants de la direction, du volume et de la composition des flux migratoires (OIM 2008), et est clairement, comme ailleurs dans le monde, l'un des principaux déterminants des migrations clandestines. Cependant, la dimension économique n'est pas le seul paramètre à prendre en compte pour expliquer les migrations en provenance de la Casamance. 


\section{Difficulté d'obtention de visa et influence des immigrés}

13 plusieurs années les poussent, le plus souvent, à avoir recours à la migration clandestine pour accomplir leur voyage. En effet, les procédures de demande de visa pour un État membre de l'Union européenne ne sont pas à la portée de tous les candidats potentiels à la migration, loin de là. Le demandeur doit fournir un justificatif d'hébergement dans le pays de destination: bail de location ou attestation d'hébergement, puis doit s'acquitter de la taxe consulaire, qui peut varier d'un consulat à un autre. Par exemple, pour un dossier de demande de visa français depuis le Sénégal, la taxe consulaire se situe entre 50 et 99 euros selon le profil du requérant (étudiant, «demandeur ordinaire») la durée (court ou long séjour) et le motif du voyage (étude, tourisme, etc.). Ce ne sont pas tant les frais de dossiers qui constituent un obstacle pour les demandeurs, mais surtout le fait qu'ils doivent certifier qu'ils disposent d'un minimum de ressources pour prouver qu'ils seront en mesure de couvrir leurs dépenses durant la durée de leur séjour. Dès lors, et face à ces procédures strictement encadrées, l'un des problèmes qui se posent est la frustration qui peut naître chez le candidat après plusieurs refus de demandes de visa. En effet, après avoir été confrontés à de nombreux refus, certains choisissent par la suite d'émigrer clandestinement, en empruntant les itinéraires terrestres ou maritimes. Pour autant, cela ne signifie pas que tous les candidats à l'émigration clandestine ont déposé au préalable une demande de visa dans leur pays d'origine avant de s'engager dans leur voyage : ils trouvent beaucoup plus simple de payer des montants compris en moyenne entre 400000 FCFA (610 euros environ) et 1200000 FCFA (1 830 euros environ) auprès de passeurs, comme l'atteste ce propos de Bachir: «avoir un visa pour l'Europe, c'est devenu impossible, si tu n'épouses pas une blanche. À moins de payer trois à quatre millions $F C F A^{9}$ mais ça, c'était impossible pour moi. La seule et unique alternative qui s'imposait à moi c'était de partir clandestinement grâce aux pirogues qui partaient depuis l'île de Djogué».

Outre la difficile obtention d'un visa, on remarque également que l'influence des immigrés (Tandian 2007), perçus comme des modèles de réussite, constitue aussi un paramètre non négligeable dans le choix de certains jeunes à prendre la mer en direction de l'Europe. Au cours de leurs vacances au pays les immigrés y construisent de belles villas, roulent dans des voitures de luxe (Nelly 1996), etc., faisant étalage de biens matériels chers acquis à l'étranger. Ils affichent de manière très ostentatoire leur richesse, ce qui a des effets puissants sur la population locale, et plus particulièrement les jeunes, comme l'évoque Hamidou, qui a tenté à deux reprises le voyage en embarquant sur une pirogue :

[...] Maintenant regarde cette autre maison en étage toute carrelée, toute propre, presque clinquante, avec son toit terrasse fleuri d'où tu vois le fleuve; elle tranche, dans le quartier, par rapport aux petites bicoques. Celle-là, je te le dis, elle existe parce que la famille a un fils là-bas de l'autre-côté qui gagne bien sa vie et envoie tous les mois de l'argent. Oh ! Ça fait longtemps qu'il a quitté ici. Lui, il a pu partir légalement quand l'Europe accueillait encore à bras ouverts ceux de leurs anciennes colonies. Il a un bon travail là-bas, en France. Il est comptable et gagne très bien sa vie, je te le dis et c'est vrai. Il a fondé une famille, et tout le monde est heureux. Il revient rarement, mais il a réussi à construire cette belle maison. C'est pour ça que j'ai essayé de partir. 


\section{De la construction d'un imaginaire migratoire à une idéalisation de l'Europe}

Les projets migratoires clandestins des jeunes de la Casamance sont influencés par la représentation qu'ils se font de l'Europe, notamment par le biais des nouvelles technologies de l'information et de la communication, en particulier les médias télévisuels et internet. Les chaînes de télévision étrangères diffusent des images qui sont loin de la réalité vécue au quotidien par les populations de Casamance. Puisque la télévision est accessible et regardée par quasiment toutes les couches sociales, cela contribue à la construction d'un imaginaire auprès des jeunes, qui en arrivent à penser que toutes les conditions pour une vie meilleure se trouvent à l'étranger, et que seul le recours à l'émigration permet d'y accéder. La migration clandestine est, de fait, alimentée par une forme d'idéalisation de l'Europe. Au cours de nos enquêtes de terrain, un constant récurrent apparaissait: la grande majorité des jeunes que nous avons interviewés s'est construit un imaginaire paradisiaque de l'Europe, comme le montrent ces propos de Moussa, chauffeur de taxi :

À chaque fois que je regarde les chaînes de télévision européennes, je ne vois jamais d'images de pauvreté comme c'est le cas ici au Sénégal. Quand tu regardes par exemple le journal télévisé, les films, les documentaires, ce sont toujours de belles choses, de beaux magasins, de belles voitures, de la publicité pour de la bonne nourriture, etc. Tout est bon là-bas, ce n'est pas comme ici où tu peux vivre durant toute une vie sans jamais pouvoir acheter une voiture par exemple. En Europe, ce n'est pas le cas, ce sont des jeunes, très jeunes même, qui vivent dans le luxe, avec appartement, voiture, voyages, et pleines d'autres choses. Nous aussi, nous avons le droit à cette vie meilleure et nous ne devons pas rester les bras croisés, il faut que nous partions, c'est seulement de cette manière qu'on peut s'en sortir ${ }^{10}$.

On remarque ainsi la prégnance de cette représentation idyllique et quasi fantasmée de l'Europe chez nombre de candidats à la migration, qui sont convaincus qu'ils trouveront un emploi dès leur arrivée en Europe et qu'ils gagneront rapidement de l'argent, ce qui reste, selon eux, impossible dans leur pays. Or, très souvent, les migrants qui réussissent leur voyage sont par la suite déçus, dans la mesure où ils constatent, une fois sur place, le fossé énorme existant entre les images telles qu'elles sont véhiculées dans les médias, et la réalité à laquelle ils sont confrontés à leur arrivée (taux de chômage élevé, travail informel, précarité, etc.).

En somme la présence du conflit armée dans la région, le chômage et la pauvreté ambiante émaillée de conditions de vie difficiles rend très difficile l'existence aux jeunes et à leur famille. Dans une telle situation où les premiers signes d'une vie qui tend à s'améliorer restent sans grand espoir et s'amenuisent, au contraire, de jour en jour, le chemin de la migration plus précisément de la migration clandestine reste la seule alternative pour bon nombre de jeunes de la Casamance. Au regard des difficultés d'obtention de visa, la migration clandestine par la route de la mer constitue une issue, il reste maintenant à trouver les moyens ou les ressources qui permettent d'effectuer ce voyage. 


\section{Les types de mobilisation des ressources pour le voyage dans la migration clandestine}

La réalisation d'un projet migratoire clandestin nécessite des ressources financières dont ne disposent pas tous les candidats. Diverses stratégies sont alors déployées par les intéressés afin de parvenir à mobiliser les sommes nécessaires. Des études ont montré par exemple l'existence de mobilisations (Gueye 2007) de type communautaire, de type familial et de type individuel dans la migration dite légale, notamment dans la vallée du fleuve Sénégal. Les mobilisations de type communautaire renvoient par exemple aux diverses stratégies mises en œuvre par tout un village pour mobiliser les ressources qui leur permettraient d'envoyer l'un des leurs à l'étranger, en espérant avoir en retour des " rémittences ». Dans le cas de la migration clandestine, au contraire, les mobilisations communautaires sont absentes, puisqu'il semblerait qu'au regard des risques d'échec que présente la migration clandestine (mort du candidat, risque d'expulsion, etc.), une communauté ou un village entier ne peut prendre la décision d'investir toutes leurs ressources dans un voyage qui se révèle être incertain. C'est l'une des raisons pour laquelle ces trois types de mobilisations ne sont pas tous présents dans la migration clandestine par la mer: on n'y trouve que les mobilisations de type individuel et/ou familiales.

\section{Les mobilisations de type individuel}

19 Par mobilisation de type individuel, nous entendons désigner les candidats qui financent leur voyage sur leurs fonds propres, même si les stratégies individuelles de mobilisation des ressources varient d'un candidat à un autre. Certains d'entre eux parviennent à épargner durant quelques années, en exerçant diverses activités dans le secteur informel. Il s'agit, pour eux, de faire l'impasse sur beaucoup de choses afin de pouvoir épargner suffisamment pour financer leur voyage.

J'ai financé mon voyage en épargnant pendant des années. À chaque fois que je fermais le soir ma cantine, je mettais de côté 3000 FCFA $^{11}$ dans un petit sac que j'avais réservé à cet effet. Ensuite à chaque fin du mois j'amenais cet argent au Crédit mutuel Sénégalais [CMS]. J'ai fait l'impasse sur beaucoup de choses. Je ne me souciais pas d'acheter de beaux habits ni de téléphone portable cher. Je n'avais qu'un seul objectif : épargner suffisamment pour aller en Europe. Une fois mon voyage réussi, je vais me tourner vers ces choses-là. Ce qui me préoccupait en ce temps-là, c'était de quitter le pays et rien d'autre ${ }^{12}$.

D'autres ont pu obtenir leurs ressources en faisant des petites activités de commerce (vente de vêtements, de chaussures et autres accessoires), de menuiserie, de mécanique, etc., l'essentiel étant de gagner de l'argent, peu importe le type d'emploi. L'autre source de mobilisation de type individuel renvoie aux prêts que peuvent contracter les candidats auprès de leur entourage, prêts qu'ils s'engagent à rembourser selon une périodicité bien déterminée. Puisque le candidat doit négocier avec son créancier la durée selon laquelle il s'engage à rembourser l'argent, il est nécessaire que s'instaure une relation de confiance entre eux. Il s'agit donc d'une forme de contrat moral qui lie les deux protagonistes, et qui engage le candidat à respecter cet accord, que son projet migratoire réussisse ou échoue. Dans ces conditions, ces prêts se font de manière générale au sein de l'entourage familial, ou auprès d'amis proches. 


\section{Les mobilisations de type familial}

21 Le voyage implique dans une certaine mesure la famille, et les mères de famille au premier chef, qui font preuve d'une grande détermination pour trouver les ressources nécessaires qui leur permettraient de financer le voyage de leur enfant. Elles sont donc fortement impliquées dans ces projets migratoires, et le plus souvent, elles vendent leurs biens ou sollicitent l'aide d'autres membres de leur entourage. Dans tous les cas, ces mobilisations sont des stratégies élaborées par les familles, dans le but d'améliorer leurs conditions de vie par des transferts d'argent provenant du candidat, s'il réussit son voyage. Que ce soit par la vente de bijoux, de bétail, ou par le biais associatif (en particulier les tontines ${ }^{13}$ ), de nombreuses mères de famille ont pu réussir à financer le voyage de leur enfant. Certaines d'entre elles, ont pu ainsi, une fois leur part de tontine relevée, soutenir leurs enfants dans leur projet migratoire comme le montrent ces propos d'Aida :

Lorsque mon tour de tontine est arrivé, j'ai pris une partie de cet argent que j'ai mis avec l'argent de mon mbotay ${ }^{14}$ pour le donner à mon fils pour qu'il parte gagner sa vie comme les autres jeunes de sa génération. J'étais consciente du danger des voyages en pirogue, parce qu'au journal télévisé de 20 heures, on nous montre souvent des naufrages de pirogues de clandestins ou des opérations de sauvetage dans les eaux méditerranéennes. Cependant, les conditions de vie dans lesquelles nous vivons depuis plusieurs années sont très difficiles. Nous avons tout fait, vraiment tout, pour nous en sortir, du commerce surtout, au regard du manque d'emploi dans le pays, c'est la seule activité qui peut nous rapporter de l'argent. En plus, beaucoup de jeunes du quartier ont été soutenus par leur famille pour qu'ils aillent tenter leur chance en Europe. Ces jeunes reviennent au bout de quelques années pour prendre soin de leurs parents et de leur famille ${ }^{15}$.

Cette stratégie fonctionne comme une forme de don et de contre-don (Mauss 2012), pour emprunter le terme de l'anthropologue Marcel Mauss. Il existe toujours des attentes de la part de ces mères de famille, dans la mesure où cet argent issu des tontines est le plus souvent épargné durant des mois, voire des années.

L'examen des différents types de mobilisation dans la migration clandestine montre que les ressources nécessaires pour financer le voyage proviennent d'horizons divers: épargne, tontines, mbotays, revenus issus de la vente des biens des parents, prêts... Diverses tactiques et stratégies sont ainsi mises en œuvre par les candidats et leur famille pour réaliser leur ambition migratoire avec une grande détermination permettant, d'une part d'inscrire ces mobilisations dans le registre d'une économie de survie et d'autre part de dégager une caractéristique nouvelle de la tontine: alors qu'à l'origine la tontine traduit la nécessité et le besoin de constituer une épargne et de renforcer les liens de solidarité entre les femmes ${ }^{16}$, nous pouvons affirmer, en Casamance, l'existence, de tontines spécifiquement constituées pour financer les voyages dans la migration clandestine s'effectuant particulièrement par la route de la mer.

\section{Conclusion}

Pour conclure, nous pouvons affirmer que la détérioration du tissu économique et social en Casamance, ainsi que la situation d'instabilité liée au conflit se déroulant dans cette région, favorise la propension des jeunes à émigrer en direction de l'Europe, dans le but 
d'améliorer leurs conditions de vie et celles de leur famille. Plusieurs facteurs objectifs et subjectifs font que la migration devient de plus en plus la seule alternative crédible pour les populations locales, et en particulier pour les jeunes. Face aux difficultés rencontrées par ces individus pour obtenir un visa, leur projet migratoire se transforme dans la plupart des cas en un voyage clandestin vers l'Europe. En outre, tant que les mirages télévisuels de vie riche et facile, qui font rêver les jeunesses de Casamance, ne seront pas compensés par des actions en faveur du développement de cette région, les jeunes continueront à émigrer clandestinement, quelle que soit la dangerosité de ces voyages.

Enfin, l'analyse des différents types de mobilisations servant à financer ce projet migratoire montre en filigrane que diverses stratégies sont déployées, aussi bien par les familles que par les candidats à la migration, au prix d'énormes sacrifices et de privations, parfois sur des périodicités très longues. Il est également important de souligner le fait que les candidats et leur famille sont conscients des dangers que présentent ces voyages, mais que leurs conditions de vie et l'image qu'ils se font de la vie en Europe les poussent à effectuer une telle aventure. Il n'est donc pas anodin de constater qu'en dépit des mesures répressives mises en place par les pays de destination, en particulier les États membres de l'UE qui renforcent le contrôle à leurs frontières extérieures, les candidats continuent d'affluer par centaines, à intervalles plus ou moins réguliers, vers les enclaves espagnoles de Ceuta et Melilla. Les mouvements migratoires continuent donc à s'intensifier depuis la Casamance, mais également depuis d'autres régions du Sénégal (Dakar, Saint-Louis et Mbour). Dès lors, la migration clandestine tend à dépasser la seule recherche d'un emploi et de meilleures conditions de vie, pour englober des valeurs sociales, traduisant ainsi tout le sens de l'expression wolof «barça wala barzakh $» .{ }^{17}$

\section{BIBLIOGRAPHIE}

Bourdieu Pierre (1994), Raisons pratiques : Sur la théorie de l'action, Paris, Seuil.

Engbersen, Godfried (1999), Sans-papiers. Les stratégies de séjour des immigrés clandestins, Actes de la recherche en sciences sociales, vol. 129, p. 26-38.

Gueye, Doudou Dieye (2007), Les mobilisations pour le départ migratoire, Migrations Société, vol. 19, n 109, janvier-février, p. 11-26.

Marut Jean-Claude (2010), Le conflit de Casamance. Ce que disent les armes, Paris, Karthala.

Mauss, Marcel (2012), Essai sur le don. Forme et raison de l'échange dans les sociétés archaïques, Paris, Presses universitaires de France.

Nelly Robin (1996), Atlas des migrations ouest africaines vers l'Europe de 1985 à 1993, Paris, Institut de recherche pour le développement.

Niang, Abdoulaye (2000), Les associations en milieu urbain Dakarois : classification et capacités développantes, Afrique et développement, vol. XXV, $\mathrm{n}^{\circ}$ 1-2, p. 99-159. 
OIM (2008), World migration report 2008: Managing labour mobility in the evolving global economy, Genève, p. 173-199.

Perruchoud, Richard (2007), Glossaire de la Migration, Organisation internationale pour les migrations, Genève.

Pian Anaïk (2009), Aux nouvelles frontières de l'Europe. L'aventure incertaine des Sénégalais au Maroc, Paris, La Dispute.

République du Sénégal, ministère de l'Économie, des finances et du plan, Agence nationale de la statistique et de la démographie, Recensement général de la population et de l'habitat, de l'agriculture et de l'élevage, 2013.

Tandian Aly (2007), Barça ou barsaax (Aller à Barcelone ou mourir) : le désenchantement des familles et des candidats à la migration, Diasporas, Histoire et Sociétés, n 9, p. 124-137.

\section{NOTES}

1. Ce sont deux petites parties de la côte marocaine qui bordent la Méditerranée. Ceuta (environ 84000 habitants), située à $49 \mathrm{~km}$ à l'Est de Tanger sur la côte, compte $18 \mathrm{~km}$ carrés ; comme Tanger elle est donc au bord du détroit de Gibraltar, un détroit de quatorze kilomètres de large qui sépare le Maroc de l'Espagne et donc le continent africain du continent européen. Melilla quant à elle (environ 80000 habitants) ne fait que $12 \mathrm{~km}$ carrés et est située nettement plus à l'Est sur la côte marocaine près de la ville marocaine de Nador à environ $400 \mathrm{~km}$ par la route de Tanger et du détroit de Gibraltar. Ce qui fait la grande particularité de ces deux minuscules territoires c'est que bien que le Maroc les revendique depuis 1956, ils sont reconnus par le Droit international comme faisant partie de l'Espagne qui leur a donné le statut de villes autonomes. Comme l'Espagne fait partie de l'Union Européenne, Ceuta et Melilla sont donc des enclaves en territoire marocain qui font partie de l'UE.

2. Cet article s'inscrit dans le cadre de la préparation d'un doctorat sur Les dynamiques migratoires clandestines en Casamance (région du sud du Sénégal): de l'analyse du contexte à la micro-économie des familles. Pour les besoins de l'étude nous suivons dans la durée dix-sept candidats à la migration clandestine et cinq familles. Basée essentiellement sur une approche combinant entretiens, récits de vie et observations, l'enquête se déroule depuis 2013 en Casamance. Au total, dix-sept entretiens semi-directifs et dix-sept récits de vie ont été effectués avec des candidats et leur famille. Les entretiens ont été effectués en wolof et traduits ensuite en français.

3. Cérémonie traditionnelle de circoncision en milieu Diola où l'on regroupe des centaines de jeunes de tout âge provenant de plusieurs villages de la Casamance. Pendant toute la période de circoncision marquant leur entrée dans le «bois sacré ", les jeunes sont isolés dans la forêt avec les anciens qui s'occupent de leur initiation. La sortie du «bois sacré » donne lieu à des cérémonies festives mêlant danses et chants traditionnels en présence des initiés et de leurs familles.

4. Selon l'UNESCO, «le kankourang est un initié qui porte un masque fait d'écorce et de fibres rouges d'un arbre appelé faara. Il est vêtu de feuilles et son corps est peint de teintures végétales. Il est associé aux cérémonies de circoncision et aux rites initiatiques. »

5. Un premier cessez-le-feu le 31 mai 1991, un deuxième le 26 décembre 1999, un troisième le 30 décembre 2004. Des accords de paix ont été aussi signés en 1992, 2001 et 2004.

6. Les maisons en banco sont construites à partir d'un mélange d'argile, de sable, de paille, de bouse de vache, d'eau... Elles se distinguent des maisons faites à base de ciment, de béton et de fer. 
7. Terme wolof utilisé par les candidats pour désigner les pirogues qui leur servent de moyens de transport au cours de leur voyage.

8. Souleymane, 28 ans, vendeur de fripes, Boucotte, Ziguinchor.

9. L'équivalent respectivement de 4570 euros et 6100 euros environ. Ce montant permet de justifier que le candidat dispose de ressources suffisantes pour couvrir l'ensemble des frais liés à son séjour dans le pays d'accueil.

10. Moussa, 32 ans, chauffeur de taxi, Ziguinchor.

11. L'équivalent de $4,50 €$.

12. Ansoumane, 30 ans, commerçant, Ziguinchor.

13. La tontine est un système d'épargne rotative nécessitant des cotisations de la part de chaque participant ou participante. À tour de rôle chaque souscripteur prélève la part totale de la mise jusqu'à ce qu'on atteigne la totalité des participants.

14. «Le mbotay regroupe des personnes ayant un potentiel affinitaire à développer entre eux et dont la vocation affichée est de consolider l'amitié et les liens de solidarité existant entre les membres, lesquels sont d'ailleurs le plus souvent déjà imprégnés des liens sociaux fondés sur des relations de voisinage ou de parenté »: Niang, Abdoulaye, "Les associations en milieu urbain Dakarois : classification et capacités développantes ", Afrique et développement, vol. XXV, 2000, n - 1-2, p. 99-159, p. 109.

15. Aida Badji, 51 ans, teinturière à Ziguinchor.

16. Bien vraie qu'on remarque une plus grande présence des femmes, il est nécessaire de souligner que les tontines ne sont pas uniquement l'apanage des femmes. Elles concernent aussi les hommes même s'ils représentent un faible pourcentage.

17. Littéralement «Barcelone ou le purgatoire ».

\section{RÉSUMÉS}

Depuis quelques années, les enclaves de Ceuta et Melilla constituent le point d'entrée de milliers de migrants en provenance pour la plupart des pays subsahariens et de l'Afrique de l'ouest. Au Sénégal, l'immigration clandestine a pris des proportions inquiétantes dans certaines régions du pays notamment en Casamance où de nombreux jeunes ont emprunté les chemins de la migration clandestine. À partir d'enquêtes effectuées depuis 2013 en Casamance, cet article examine les déterminants et les types de mobilisations de ressources impliquées par ces voyages.

Since a few years, the enclaves of Ceuta and Melilla constitutes the entry point of thousand migrants in origin for the most part of the sub-saharan countries and of western Africa. In Senegal, the illegal immigration took disturbing proportions in certain regions of the country in particular in Casamance where numerous young people followed the paths of the illegal migration. From inquiries made since 2013 in Casamance, this article examines determiners and kinds of mobilizations of resources involved by these journeys. 
AUTEUR

ABDOULAYE NGOM

Doctorant en Sociologie

UMR 7367 Dynamiques Européennes, CNRS / Université de Strasbourg 